\title{
MORE PARACOMPACT BOX PRODUCTS
}

\author{
JUDY ROITMAN
}

\begin{abstract}
We show that if there is no family of cardinality less than c which dominates $\omega_{\omega} \omega$, then the box product of countably many compact first-countable spaces is paracompact; hence the countable box product of compact metrizable spaces is paracompact if $2^{\omega}=\omega_{2}$. We also give classes of forcing extensions in which many box products are paracompact.
\end{abstract}

0. Introduction. In the last six years there have been many proofs showing that various classes of countable box products were consistently paracompact. The methods of these proofs are two: the tree argument used by van Douwen, and the stratification argument used by everyone else. Here we generalize the latter technique to show:

THEOREM 0. (a) If no family of cardinality less than c dominates $\omega_{\omega} \omega$, then the box product of countably many compact first-countable spaces is paracompact.

(b) If $\mathrm{c}=\omega_{2}$, then the box product of countably many compact metrizable spaces is paracompact.

We then generalize the method further to show that a simplified version of the principle implicit in [Ro] also implies that the box product of countably many compact first-countable spaces is paracompact. From this we find a wide class of forcing extensions in which various box products are paracompact, thus pointing out where not to look in trying to give a negative answer to the following open questions:

A. Is $\square^{\omega}(\omega+1)$ paracompact?

B. Does the existence of a $\lambda$-scale for some $\lambda$ imply that the countable box product of compact first-countable spaces is paracompact?

Thanks are due to William Mitchell and Saharon Shelah who pointed out that the proofs in this paper did more than they were expected to do.

1. Preliminaries. All spaces are assumed to be Hausdorff. A space $X$ is paracompact iff every open covering of $X$ has a locally finite refinement. The letters $f, g, h$ are reserved for functions from $\omega$ to $\omega ; A, B$ for infinite subsets of $\omega$.

Let $I$ be an index set, $X_{i}$ a topological space for each $i \in I$. Then the box product $\square_{i \in I} X_{i}$ consists of all points in $\prod_{i \in I} X_{i}$ under the topology whose

Received by the editors February 6, 1978 and, in revised form, May 1, 1978.

AMS (MOS) subject classifications (1970). Primary 54D20; Secondary 04A20. 
basis consists of all boxes $u=\Pi_{i \in I} u_{i}$, where each $u_{i}$ is open in $X_{i}$. If $x=\left\langle x_{i}\right.$ : $i\langle\omega\rangle$ and $y=\left\langle y_{i}: i\langle\omega\rangle\right.$ are in $\square_{i \in \omega} X_{i}$, we say $x \equiv y$ iff $\left\{i: y_{i} \neq x_{i}\right\}$ is finite. $\nabla_{i \in \omega} X_{i}$ is the quotient topology on the equivalence classes of $\square_{i \in \omega} X_{i}$ generated by $\equiv$.

The connection between $\square$ and $\nabla$, and the reasons $\nabla$ is nice to work with, are made clear by the following theorem of Kunen:

THEOREM 1 (Kunen). (a) If each $X_{i}$ is compact, then $\square_{i \in \omega} X_{i}$ is paracompact iff $\nabla_{i \in \omega} X_{i}$ is paracompact.

(b) $G_{\delta}$ 's in any $\nabla_{i \in \omega} X_{i}$ are open; hence if each $X_{i}$ is regular, the $G_{\delta}$ 's of $\nabla_{i \in \omega} X_{i}$ are clopen, and the space is 0 -dimensional.

(c) $\nabla_{i \in \omega} X_{i}$ is paracompact iff every open cover has an open disjoint covering refinement (such a space is called ultraparacompact).

Because of Kunen's theorem, for the rest of this paper we work with $\nabla$, and will prove that under various circumstances $\nabla_{i \in \omega} X_{i}$ is paracompact, where each $X_{i}$ is regular. The reader can then conclude that if each $X_{i}$ satisfies the desired hypotheses and is also compact, then $\square_{i \in \omega} X_{i}$ is paracompact as well.

The connection between $\nabla$ and the structure of $\omega_{\omega}$ is made clear by the following notation and definitions.

Suppose, for each $i \in \omega, X_{i}$ is first countable. For each $x_{i} \in X_{i}$, we fix $u_{x_{i}}$ a function from $\omega$ such that $\left\{u_{x_{i}}(j): j \in \omega\right\}$ is a descending neighborhood basis for $x_{i}$. Then given $f: \omega \rightarrow \omega$ and $x \in \nabla_{i \in \omega} X_{i}$, we define $u_{x_{f} f}=\nabla_{i \in \omega} u_{x_{i}}(f(i))$.

Given $f, g, A$, we say $f<g$ on $A$ iff $\{i \in A: f(i) \nless g(i)\}$ is finite. Then the following fact is immediate:

FACT 2. If $x, y \in \nabla_{i \in \omega} X_{i}$ and $f, g \in{ }^{\omega} \omega$, then letting $C=\left\{i: u_{x_{i}}(f(i)) \cap\right.$ $\left.u_{y_{1}}(g(i))=\varnothing\right\}, C$ is infinite iff $u_{x_{f}} \cap u_{y, g}=\varnothing$; and if $u_{x, f} \cap u_{y, g}=\varnothing$ and $h \nless f$ on $C$, then $u_{x, h} \cap u_{y, g}=\varnothing$.

The careful reader will observe in the preceding sentence a slight confusion of events in $\Pi X_{i}$ and $\nabla X_{i}$. Such confusions will continue to occur for the sake of readability.

$\mathscr{F} \subset{ }^{\omega} \omega$ is a dominating family iff for every $f \in \omega_{\omega}$ there is some $g \in \mathscr{F}$, $g<f$ on $\omega$. A $\lambda$-scale is a dominating family well ordered by $<$ of order type $\lambda$. The space $\omega+1$ is the order type $\omega+1$ under the interval topology.

We write $\mathscr{D}_{\alpha} \neg \mathscr{Q}, \alpha<\lambda$, iff $\mathscr{D}=\cup_{\alpha<\lambda} \mathscr{D}_{\alpha}$ and $\alpha<\beta \Rightarrow \mathscr{D}_{\alpha} \subseteq \mathscr{D}_{\beta}$.

2. Combinatorial proofs. In this section we state two combinatorial principles and prove that they establish paracompactness.

Definition 3. Let $\mathscr{F} \subset{ }^{\omega} \omega, \mathbb{Q} \subset \mathcal{P}(\omega)$. We say $\mathscr{F}$ is cofinal on $\mathbb{Q}$ iff for every $f$ there exists an $A \in \mathbb{Q}$ and a $g \in \mathscr{F}$ such that $g>f$ on $A$.

The principle $(*)$ says:

$$
\text { If }|\mathscr{F}|,|\mathscr{Q}|<\mathbf{c} \text {, then } \mathscr{F} \text { is not cofinal on } \mathbb{Q} \text {. }
$$

FACT 4. (*) is equivalent to the assertion that there is no dominating family of cardinality $<\mathbf{c}$. 
Proof. One direction is trivial. We prove the not-quite-so-trivial direction. Suppose (*) is false via $\mathscr{F}, \mathbb{Q}$ and we have some $g \in \omega_{\omega}$. We may assume that each function in $\mathcal{F}$ is increasing. There exists an $f \in \mathcal{F}$, and an $A \in \mathcal{Q}$, with $f>g$ on $A$. Suppose $A=\left\{a_{0}, a_{1}, \ldots\right\}$ in increasing order. Define $h$ to be constantly equal to $f\left(a_{n+1}\right)$ on the interval $\left[a_{n}, a_{n+1}\right)$. Then $h>g$ on $\omega$ and there can be at most $|\mathscr{F}| \cdot|\Theta|<\mathbf{c}$ such $h$ 's.

Notes on $(*)$. 1. (*) $\Rightarrow$ there is no $\lambda$-scale, for $\lambda<\mathbf{c}$.

2. By Fact $4,(*)$ is true if there is a c-scale and $c$ is regular, hence under MA.

By Fact 4 and Theorem 1, Theorem 0 (a) will be proved by the following

THeOReM 5. (*) implies that $\nabla_{i \in \omega} X_{i}$ is paracompact if each $X_{i}$ is first countable, Lindelöf, and regular.

Proof. By a well-known theorem of Arhangel'skii, each $X_{i}$ has cardinality $\leqslant$ c, hence $X=\nabla_{i \in \omega} X_{i}$ has cardinality c. We construct a refinement of a given cover $\mathcal{Q}$ by an induction of length $\mathrm{c}$. Suppose we have assigned $X$ some well ordering of type $c$ and have already covered the first $\beta$ elements of $X$ by clopen sets, where this covering $\mathscr{U}_{\beta}$ refines $\mathcal{Q},\left|\mathcal{U}_{\beta}\right| \leqslant|\beta|<\mathbf{c}$, and each $v \in \mathcal{Q}_{\beta}$ is of the form $\bigcap_{i \in \omega} u_{y, f_{i}}$, where $\left\{f_{i}: i \in \omega\right\}$ is an increasing sequence. Let $x$ be the next point we have to cover. If $x$ is already covered, we simply let $\mathcal{U}_{\beta+1}=\mathcal{Q}_{\beta}$. Otherwise, for each $v \in \mathcal{U}_{\beta}$ we can find a $g_{v}$ so that $u_{x, g_{i}} \cap v=\varnothing$. Hence, where $v=\bigcap_{i \in \omega} u_{y f_{i}}$, we can find an $i_{v}$ so that

$$
u_{x, g_{i}} \cap u_{y, f_{i}}=\varnothing
$$

(this is because the $f_{i}$ 's are increasing). Hence by Fact 2,

$$
A_{v}=\left\{k: u_{x_{k}}\left(g_{v}(k)\right) \cap u_{y_{k}}\left(f_{i_{r}}(k)\right)=\varnothing\right\}
$$

is infinite. By hypothesis we can find a single $h$ so that for every $v \in \mathcal{Q}_{\beta}$, $g_{v} \nless h$ on $A_{v}$. Hence by Fact $2, u_{x, h} \cap v=\varnothing$ for all $v \in \mathcal{Q}_{\beta}$. Let $h_{i}$ be an increasing sequence, $h_{0}=h$. We let $\mathscr{Q}_{\beta+1}=\mathscr{Q}_{\beta} \cup\left\{\cap_{i \in \omega} u_{x, h_{i}}\right\}$.

Theorem 0 (b) then follows from Theorem 5 by the following theorem of van Douwen: if there is a $\lambda$-scale for some ordinal $\lambda$, then the countable box product of compact metrizable spaces is paracompact.

Proof of 0 (b). Assume $\mathbf{c}=\omega_{2}$. If ( $*$ ) is false, then there is a dominating family of size $\omega_{1}$, which is easily seen to contain an $\omega_{1}$-scale. So we are either in the situation of Theorem 5, or the hypothesis of van Douwen's theorem.

(*) is a statement about $\omega_{\omega}$ and the power set of $\omega$; no topological spaces are mentioned. (*) is also implicitly a statement about $\nabla \omega(\omega+1)$ : it says that: $(\dagger)$ there is a basis $\mathscr{B}$ so that the union of less than $c$ sets from $\mathscr{B}$ is closed. The proof of Theorem 5 is also a proof that $(\dagger)$ holds for any $\nabla_{i \in \omega} X_{i}$, if each $X_{i}$ is first countable, Lindelöf, and regular.

For our next principle we are not so lucky. We need to know about more than the structure of $\omega_{\omega}$ and the power set of $\omega$. We want a method of proof that stratifies a space $X$ under consideration so that we may simultaneously 
cover each layer. Therefore we do not have a general combinatorial principle, but one which depends on the particular space.

Definition 6. Let $X=\nabla_{i \in \omega} X_{i} \cdot(* *)_{X}$ is the statement that for some ordinal $\lambda$ there exist $X_{\alpha} \nearrow X, \alpha<\lambda ; \mathbb{Q}_{\alpha} \nearrow \mathscr{P}(\omega), \alpha<\lambda ; \bigodot_{\alpha} \nearrow \omega \omega, \alpha<\lambda$; and $\mathscr{F}=$ $\left\{f_{\alpha}: \alpha<\lambda\right\} \subset \omega^{\omega}$ such that

(1) $\alpha<\beta \Rightarrow f_{\beta} \in C_{\alpha}$,

(2) $f \in \mathcal{C}_{\alpha}, A \in \mathbb{Q}_{\alpha} \Rightarrow f \ngtr f_{\alpha}$ on $A$,

(3) $f, g \in \mathcal{C}_{\alpha} \Rightarrow f+g \in \bigodot_{\alpha}$,

(4) $X_{\alpha}$ is Hausdorff under the topology generated by all $u_{x, f}$, where $x \in X_{\alpha}$, $f \in \mathcal{C}_{\alpha}$,

(5) if $x, y \in X_{\alpha}, f, g \in \mathcal{C}_{\alpha}$, and $u_{x, f} \cap u_{y, g}=\varnothing$, then $\left\{i: u_{x_{i}}(f(i)) \cap u_{y_{i}}(g(i))\right.$ $=\varnothing\} \in \mathbb{Q}_{\alpha}$.

Notes on $(* *)_{X} .1$. Clauses 3,4 , and 5 simultaneously stratify $X, \omega_{\omega}$, and $\mathcal{P}(\omega)$ so that if we only know about the functions and sets on the $\alpha$ th layer, we already know that $X_{\alpha}$ is Hausdorff. Clauses 1 and 2 connect this stratification with a sequence of functions which may not be dominating, but which no level can dominate.

2. $(* *)_{X}$ is a simplified version of the combinatorics in [Ro].

3. (*) implies (**) $)_{X}$ if $X=\nabla_{i \in \omega} X_{i}$ and each $X_{i}$ is first countable, Lindelöf, and regular. Also the existence of a $\lambda$-scale for some $\lambda$ implies $(* *)_{X}$ if $X=\nabla^{\omega}(\omega+1)$.

4. As the complication of Definition 6 and the anthropomorphism of Note 1 indicate, $(* *)_{X}$ is designed to tell us when a forcing extension makes box products paracompact.

THEOREM 7. Let $X=\nabla_{i \in \omega} X_{i}$, where each $X_{i}$ is regular and first countable. Then $(* *)_{X}$ implies that $X$ is paracompact.

Proof. Again we proceed by induction, this time on the $\lambda$ of Definition 6 . Let $\mathcal{U}$ be an open covering of $X$. Suppose at stage $\alpha<\lambda$ we have covered a subset of $\cup_{\beta<\alpha} X_{\beta}$ by a disjoint refinement $\mathscr{U}_{\alpha}$ of $\mathscr{U}$, where each $v \in \mathcal{Q}_{\alpha}$ is clopen and of the form $\bigcap_{n \in \omega} u_{x, n f}$ for some $x \in \cup_{\beta<\alpha} X_{\beta}, f \in \cup_{\beta<\alpha} \bigodot_{\alpha}$ (and $n f(j)=n \cdot f(j)$ for all $j \in \omega$ ). Let $\mathcal{V}$ be the collection of all $u_{y, g}$, for $y \in X_{\alpha}, g \in \mathcal{C}_{\alpha}$. By $(* *)_{X}, \mathcal{V}$ separates points in $X$. If $y \in X_{\alpha}$ has not been covered yet, let $h_{y}>f_{\alpha}$ where $u_{y, h_{y}}$ refines a neighborhood in $\mathcal{Q}, h_{y} \in \mathcal{C}_{\alpha+1}$, if such an $h_{y}$ exists. (The problem is that no candidate for $h_{y}$ may be in $\bigodot_{\alpha+1}$.) If $h_{y}$ exists, let $u_{y}=\bigcap_{n \in \omega} u_{y, n h_{y}}$ and let $\mathscr{U}_{\alpha+1}=\mathscr{U}_{\alpha} \cup\left\{u_{y}: y \in X_{\alpha}-\cup \mathcal{U}_{\alpha}\right.$ and $h_{y}$ is defined $\}$. Clauses 2,4 , and 5 of the definition guarantee that $\mathcal{U}_{\alpha+1}$ is a disjoint refinement. Clause 3 says that each $n h_{y}$ is in $\bigodot_{\alpha+1}$. That every $y$ is eventually covered follows from 1,3 , and the fact that $e_{\alpha} \nearrow{ }^{\omega} \omega$.

3. Forcing extensions. In what models of set theory do (*) and (**) ${ }_{X}$ hold? We partially answer this question with two propositions, the first a simple criterion for $(*)$, and the second a criterion for $(* *)_{X}$. We give examples in each category so the reader will know what we are talking about. 
First, some definitions. Let $\mathbf{B}$ be a complete Boolean algebra. $\mathbf{B}$ has $\kappa$-cc iff every set of mutually incompatible elements has cardinality $<\kappa$. Note that $\kappa<\lambda$ and $\mathbf{B}$ has $\kappa$-cc $\Rightarrow \mathbf{B}$ has $\lambda$-cc. The density of $\mathbf{B}, d(\mathbf{B})$, is the least cardinality of a dense partial order in $\mathbf{B}$.

$\mathbf{B}$ has cofinality $\delta$ iff there is a sequence of algebras $\mathbf{B}_{\alpha} \nearrow \mathbf{B}, \alpha<\lambda$, where $\operatorname{cf}(\lambda)=\delta$ and each $\mathbf{B}_{\alpha}$ is a complete proper subalgebra of $\mathbf{B}$. Note that the cofinality of $\mathbf{B}$ is not unique.

Let $V$ be a model of set theory, $\mathbf{B} \in V$, and $\left\{\mathbf{B}_{\alpha}: \alpha<\lambda\right\} \in V$ be a witness that $\mathrm{B}$ has cofinality $\delta$. We define $V_{0}=V$, and $V_{\alpha}=V^{\mathbf{B}_{\alpha}} \subset V^{\mathbf{B}}$ for $\alpha>0$. A function $f$ in $V^{\mathbf{B}}$ is free over $V_{\alpha}$ iff for every $A, g \in V, f \nless g$ on $A$. Otherwise $f$ is dominated by $V_{\alpha} . V^{\mathbf{B}}$ is dominated by $V_{\alpha}$ iff every $f$ in $V^{\mathbf{B}}$ is dominated by $V_{\alpha}$. Otherwise $V^{\mathbf{B}}$ is free over $V_{\alpha}$.

A peculiar aspect of forcing is that $V$ often knows the size of the continuum in $V^{\mathbf{B}}$. That is, there is usually a $\kappa \in V$ so that $V \vDash \kappa=\mathrm{c}^{V^{\mathbf{B}}}$. Call this cardinal $\mathbf{c}^{\mathbf{B}}$.

Lemma 8. Suppose $V \vDash\left[\mathbf{B}\right.$ has cofinality $\mathbf{c}^{\mathbf{B}}$ and $V^{\mathbf{B}}$ is free over all $\left.V_{\alpha}\right]$. Then (*) holds in $V^{\mathbf{B}}$.

Proof. If (*) fails, there is a dominating family which is contained in some $V_{\alpha}$, contradicting the freedom of $V^{\mathbf{B}}$.

EXAMPLES. 1. Any direct iterated $\omega_{1}$-cc B with cofinality $d(\mathbf{B})^{\omega}$.

2. Mixing things up: e.g. $\mathrm{CH}$ is true in each $V_{\alpha}, \delta=\omega_{2}$, and each $\mathbf{B}_{\alpha}$ is a product or iteration of two algebras, one adding at least one free function, the other adding no new reals. Mathias and Laver forcing are examples.

LEMMA 9. Suppose $V \vDash\left[\mathbf{B}\right.$ has $\kappa^{+}-c c$ and cofinality $\delta \geqslant \kappa^{+} ; V^{\mathbf{B}}$ is free over all $V_{\alpha}$ ]. Then $(* *)_{X}$ holds in $V^{\mathbf{B}}$ for every $X=\nabla_{i \in \omega} X_{i}$ where each $X_{i}$ is regular, first countable with weight $\leqslant \delta$.

Proof. The point of the hypothesis on $\delta$ is that we can then repeat the construction of [Ro] to stratify $X$ into $X_{\alpha}$ 's. A sketch of the construction is:

To each $X_{i}$ we associate $L_{i}$, the lattice of basic open sets. By hypothesis, each $L_{i}$ is small enough so any countable descending chain is an element of some $V_{\alpha}$. We identify a point in $X_{i}$ with some countable descending chain converging to it. Then $\left(X_{i}\right)_{\alpha}$ is the collection of such chains which are elements of $V_{\alpha} ; X_{\alpha}=V_{\alpha} \cap \nabla_{i \in \omega}\left(X_{i}\right)_{\alpha}$.

${ }^{\omega} \omega \cap V^{\mathbf{B}}$ and $\mathcal{P}(\omega) \cap V^{\mathbf{B}}$ are already stratified by the $V_{\alpha}$ 's, and this natural stratification, together with the $X_{\alpha}$ 's as above, makes clauses 3,4 , and 5 of $(* *)_{X}$ true. Because $V^{\mathbf{B}}$ is free, we can find an $\mathscr{F}$ satisfying clause 2; we can then skip up the levels to satisfy 1 .

EXAMPLES. 1. Any direct $\omega_{1}$-cc extension with uncountable cofinality.

2. $\mathbf{B}=\mathbf{C} \times \mathbf{D}, \mathbf{C}$ stratifies so that it is free over each $V^{\mathbf{C}_{\alpha} \times \mathbf{D}}=V_{\alpha}$, and every real is in some $V_{\alpha}$. Candidates for such C's are $\omega_{1}$-cc algebras, and for the associated D good candidates are Solovay, Sacks, or Silver forcing which 
all have the property that new functions in $\omega_{\omega}$ are dominated by old functions.

3. $\mathbf{B}$ is an iteration $\mathbf{C} * \mathbf{D}$, and defining $\mathbf{D}_{\alpha}=\left\{p: V^{\mathbf{C}_{\alpha}} \vDash p \in \mathbf{D}_{\alpha}\right\}$, each real occurs in some $V_{\alpha}=V^{\mathbf{C}_{\alpha} \times \mathbf{D}_{\alpha}}$ and $V^{\mathrm{C}}$ is free over each $V_{\alpha}$. The same candidates for $\mathbf{C}$ and $\mathbf{D}$ in 2 are candidates here, although care must be taken so that $\mathbf{C}_{\alpha} \times \mathbf{D}_{\alpha} \nearrow \mathbf{B}$.

Looking at these and other examples, the following questions occur:

C. Must reals be added to destroy paracompact box products? (Yes if, say, (*) or "there is a $\lambda$-scale" hold in the ground model. What about other cases?)

D. Can an $\omega_{1}$-cc extension by itself destroy paracompact box products?

\section{BIBLIOGRAPHY}

[A] A. V. Arhangel'skii, On the cardinality of bicompacta satisfying the first axiom of countability, Dokl. Akad. Nauk SSSR 187 (1969).

[vD] E. K. van Douwen, Separation and covering properties of box products and products (to appear).

[K] K. Kunen, Box products of compact spaces (to appear).

[Ro] J. Roitman, Paracompact box products in forcing extensions (to appear).

[Ru] M. E. Rudin, The box product of countably many compact metric spaces, General Topology and Appl. 2 (1972).

[W] S. Williams, Is $\square^{\omega}(\omega+1)$ paracompact? Topology Proceedings 1 (1976).

School of Mathematics, Institute for Advanced Study, Princeton, New Jersey 80540

Current address: Department of Mathematics, University of Kansas, Lawrence, Kansas 66045 